\title{
Large-scale structures in a stratified open channel flow
}

\author{
Konstantin Borynyak ${ }^{1, *}$, and Michael Hrebtov ${ }^{1}$ \\ ${ }^{1}$ Institute of Thermophysics of SB RAS, 630090, 1 Ac. Lavrentiev ave., Novosibirsk, Russia
}

\begin{abstract}
We present the results of direct numerical simulation of stably stratified open channel flow. The simulation was conducted in a frame of reference moving with the mean flow. The chosen setup allowed us to apply temporal filtering without affecting the long-living large-scale laminar and turbulent patches to see the dynamics of the processes on their boundaries. The main goal of the paper was to investigate the vorticity balance in different parts of the flow. It was shown that boundaries between laminar and turbulent patches contain weak large-scale vortex structures sustained by the baroclinic generation.
\end{abstract}

\section{Introduction}

Stably stratified flows are known for their fascinating behaviour with large intermittent turbulent and laminar patches. The most common examples of such phenomena are hot airflow over a cooler ocean or night atmospheric boundary layer where the ground cools faster than the air above it. Stratified flows attract increasing attention in scientific community over past decade [1-3].

Stably stratified channel flows are a computationally low-cost model for studying intermittency caused by thermal stratification. Depending on the ratio between the shear and negative buoyancy the flow may become fully turbulent, laminar, or intermittent, with the dynamic alternation of turbulent and laminar spots.

There are two main regions in the stratified boundary layer: the outer region, far from the wall where the shear is low and the flow dynamics is mostly governed by gravity waves [4-5], and the inner region, close to the wall, where the shear is high and the turbulent production opposes the damping effect of negative buoyancy. The outer region is extensively studied and overall well-understood, while the inner region and its dynamics are much more complex, and less-understood, especially the dynamic equilibrium of longlived turbulent and laminar patches.

The complexity of the phenomena is in large amount of energetic interacting vortices in a strongly anisotropic environment. Not only the flow is anisotropic because of the effect of a wall, but it also becomes anisotropic in horizontal directions due to the intermittency effects. Such highly dynamic configuration is very hard to grasp as a whole so the hope is in statistical processing of the data to simplify the group effects of many vortex interactions in some deterministic way.

* Corresponding author: borisnsk07@gmail.com 
In this short paper we investigate the processes on the boundaries between the turbulent and laminar zones with the focus on vorticity dynamics. The vorticity dynamics and especially the large-scale structures that are masked by a numerous smaller eddies generated by the shear stress at the wall may play an important role in sustaining the dynamic equilibrium in the intermittent flow. One way to make these larger structures visible is by changing the reference frame in order to stop the advection and filtering out the small-scale eddies.

\section{Computational details}

An open channel flow with a low friction Reynolds number $\left(\operatorname{Re}_{\tau}=u_{\tau} h / v=180\right.$, where $u_{\tau}$ is a friction velocity, $v$ is the kinematic viscosity and $h$ is the open channel height) was simulated. The Richardson number $\left(R i_{\tau}=\alpha \Delta T g h / u_{\tau}^{2}=240\right.$, where $\alpha$ is thermal expansion coefficient, $g$ is gravitational acceleration and $\Delta T$ is temperature difference between top and bottom boundaries) was large enough to produce an intermittent turbulent/laminar flow pattern. The Prandtl number value was equal to 0.71 .

The computational domain dimensions were $8 \pi \times 1 \times 4 \pi$ with $512 \times 50 \times 512$ grid points respectively. In longitudinal and transversal directions the grid was uniform, while in vertical direction the grid was clustered toward the wall with the grid step range: $\Delta y^{+}=0.1-5.5$.

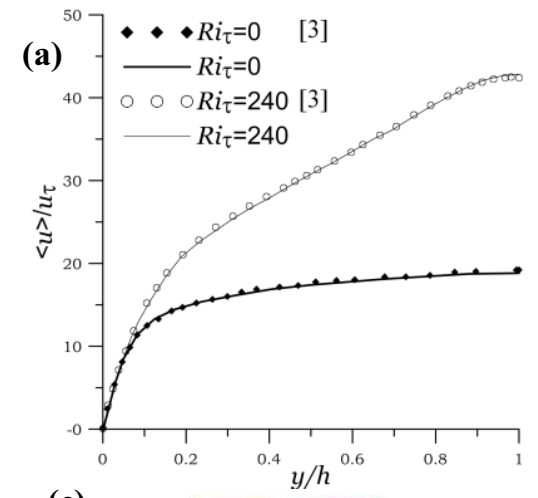

(c)

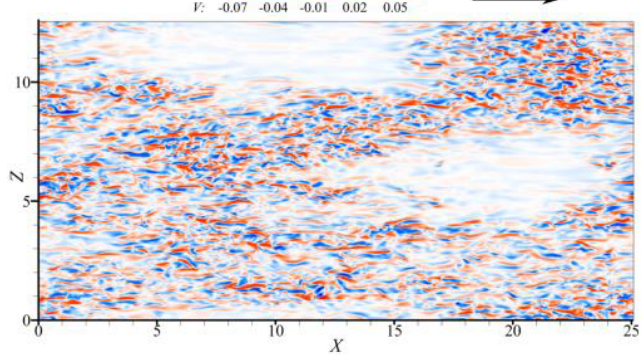

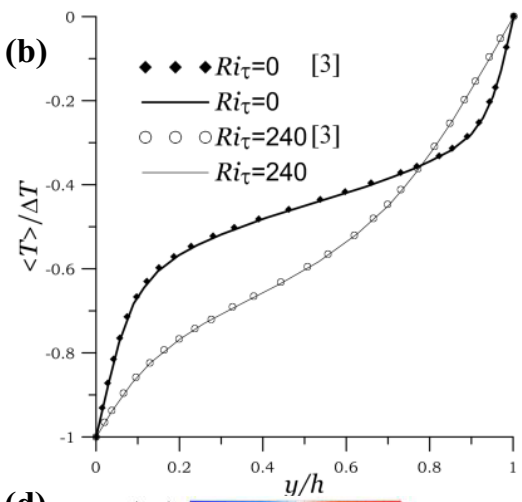

(d)
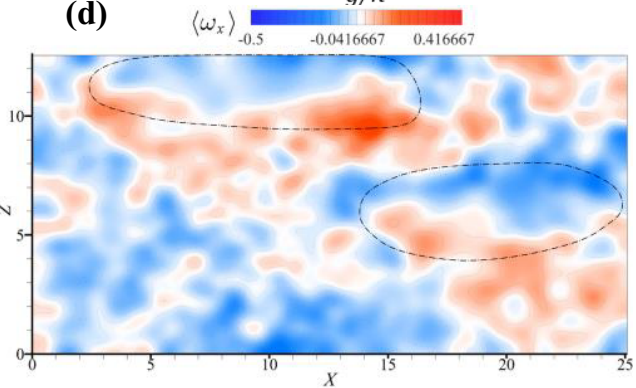

Fig. 1. Comparison of mean vertical profiles obtained in present simulation (lines) and taken from [3] (dots) for cases with stable and neutral stratification $(\mathrm{a}, \mathrm{b})$; a typical pattern of laminar and turbulent patches illustrated by vertical vorticity distribution in a cross-section $y / h=0.125$ (c); Time-filtered longitudinal vorticity distribution at the same cross-section, the lines denote the places of laminar zones in instantaneous fields (d).

We used a DNS solver based on an open-source CFD package called OpenFoam. OpenFoam has been verified for a number of scientific and engineering applications $[6,7]$. 
The Navier-Stokes equations of incompressible fluid flow with Boussinesq approximation for buoyancy force were solved numerically using the finite-volume method with second order accuracy in space and time. No-slip and free-slip conditions were set at bottom and top boundaries respectively while at other boundaries the periodic conditions were prescribed.

The simulation was conducted in a reference frame moving with the bulk velocity of the flow. This allowed us to investigate the evolution of laminar and turbulent patches as they appeared almost stationary in the chosen reference frame.

The mean velocity and temperature profiles (together with the Reynolds stress and turbulent heat flux components, not shown here) were compared with the DNS data from [3] (Fig 1a,b). The profiles show good agreement with the literature.

\section{Results and discussion}
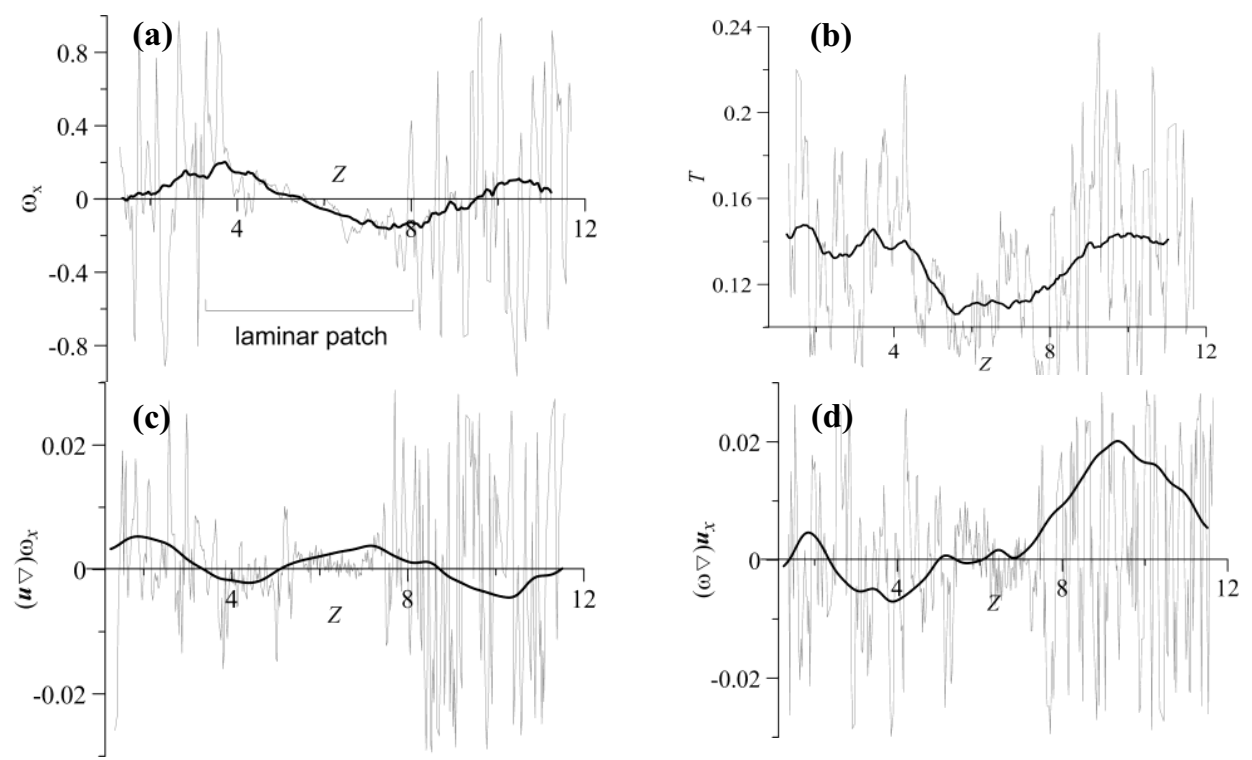

Fig. 2. Transversal profiles at $x / h=20, y / h=0.13$ for instantaneous (thin line) and time-averaged (thick line) values of: longitudinal vorticity (a); temperature (b); advection of longitudinal vorticity (c); tilting of longitudinal vorticity (d).

Fig. 1c shows a typical pattern of laminar and turbulent patches in the lower part of the channel. In our simulations the laminar spots were relatively stationary due to the chosen reference frame. This allowed us to do time-averaging of the turbulent fields without filtering out the spots structure. By using this procedure it was possible to find some weak large-scale vortex formations which were hidden behind the turbulence. Fig 1d shows the result of time averaging over $6 t_{l}\left(t_{l}=L / U_{\text {bulk }}\right.$, where $L$ is a streamwise domain length) of longitudinal vorticity component. The dotted ovals show the location of laminar spots. It is clearly seen that after time-averaging there are opposite signs of vorticity on the opposite boundaries of both laminar zones. This vorticity would induce the velocity field which lifts the flow up in the turbulent region and pulls it down in the laminar region, thus contributing to their stability.

Fig. 2a shows the transverse cross-section of the flow intersecting the laminar patch, it is clearly seen that after averaging a pattern emerge with positive and negative vorticity maximums at the boundaries between laminar and turbulent region. 
To investigate the source of this longitudinal vorticity we computed separately the components of vorticity balance equation:

$$
\frac{\partial \omega_{x}}{\partial t}=-(u \nabla) \omega_{x}+(\omega \nabla) u_{x}-\beta g \frac{\partial T}{\partial z}+\ldots
$$

From Fig $2 \mathrm{c}$ it is clearly seen that the vorticity is at least partially accumulated at the boundary due to advection. Further analysis shows that the main component of advection is longitudinal, that means that the longitudinal vorticity of the needed sign is generated somewhere upstream. The vortex bending term Fig. $2 \mathrm{~d}$ has a minimum in laminar patch and has large maximums in the turbulent areas, its direction on the boundary between the laminar end turbulent regions is opposite to the existing vorticity sign, so it would work like a sink, meaning that longitudinal vorticity is being bent in some direction. The last term in Eq. 1 is baroclinic term. This term describes the production of vorticity by buoyancy gradient. Fig $2 \mathrm{~b}$ shows the time-averaged temperature profile over the transversal crosssection. It is evident that the laminar patch is cooler than the turbulent spots thus it generate negative buoyancy. So the existing temperature gradients contribute to the vorticity distribution of Fig 1a.

One interesting effect observed in the moving reference frame is that the small velocity fluctuations inside the laminar spots are advected at lower speed than the fluctuations in turbulent patches. Thus, the small eddies from turbulent patches are frequently got into boundaries of laminar zones where they are slowed down and dissipated rapidly. This process creates a constant flow of vorticity toward the boundaries between the laminar and turbulent regions. We speculate that this vorticity flow could be connected with the described large-scale structures.

\section{Conclusion}

The simulation of stratified open channel flow in a frame of reference moving with the mean velocity allowed us to apply some statistical tools on the laminar and turbulent patches in the flow separately. Weak but large-scale vortex structures were found at the boundaries between the laminar and turbulent regions. These structures are sustained mostly by the baroclinic generation and they may take a part in the supporting of the dynamic balance between laminar and turbulent patches.

This work was supported by the Russian Science Foundation (grant №16-19-00119).

\section{References}

1. K. Yeo, B. Kim and C. Lee, J. Turbul., 10, 17 (2009)

2. M. García-Villalba, J.C. del Álamo, Phys. Fluids, 23045104 (2011)

3. P. He, S. Basu, Nonlin. Processes Geophys., 22447 (2015)

4. O. Flores, J.J. Riley, Boundary-Layer Meteorol. 139241 (2011)

5. E. Deusebio, P. Schlatter, G. Brethouwer, E. Lindborg, J. Phys.: Conf. Ser., 318 022009), (2011)

6. F. Flores, R. Garreaud, R. Muñoz, Comput. Fluids, 821 (2013)

7. D.A. Hoang, V. van Steijn, L.M. Portela, M.T. Kreutzer, C.R. Kleijn, Comput. Fluids, 8628 (2013) 